\title{
Increased 2020 impact factor for Int Journal Cardiovascular Imaging:
}

\subsection{7}

\author{
Johan H. C. Reiber ${ }^{1}$
}

Published online: 9 July 2021

(C) The Author(s), under exclusive licence to Springer Nature B.V. 2021

Dear reader,

First of all, I would like to inform you about the improvement in the Impact Factor for the International Journal of Cardiovascular Imaging: from 1.929 in 2019 to 2.357 in 2020 ! This is a great improvement, and I would like to thank all our authors, plus of course the Associate and Adjunct Editors, and the Editorial Board for their contributions to our Journal, and we look forward to a further increase in IF 2021!

For this August 2021 issue, I have selected and would like to recommend the paper entitled "Incremental improvement of diagnostic performance of coronary CT angiography for the assessment of coronary stenosis in the presence of calcium using a dual-layer spectral detector CT: validation by invasive coronary angiography" by Cheng Xu et. al. [1] from Peking Union Medical College Hospital, Chinese Academy of Medical Sciences in Beijing, China with the support of Philips Healthcare, Beijing, China and Cleveland, $\mathrm{OH}$, USA and T Leiner from the University Medical Center in Utrecht, The Netherlands. It is well known that imaging of calcium in the coronary arteries with multi-slice $\mathrm{CT}$ always results in blooming artefacts, so that the amount of calcium, but also stents always result in overestimations of its sizes. This really limits the clinical applicability.

The authors studied the use of a dual-layer spectral detector CT (SDCT), in a population of 72 consecutive patients with 122 coronary lesions, which also had calcium in the vessel wall. They compared conventional $120 \mathrm{kVp}$ images, eight virtual monoenergetic images (VMI) (70 to $140 \mathrm{keV}$ ), the effective atomic number ( $\mathrm{Z}$ effective) and iodine no water images. The results in the assessments of the narrowings in the coronary arteries were compared with invasive coronary angiography as a gold standard. The authors found that reconstruction at $100 \mathrm{keV}$ yielded optimal diagnostic performance: the sensitivity, specificity, PPV, NPV and diagnostic accuracy to identify stenoses $>70 \%$ were $78 \%$, $98 \%, 93 \%, 91 \%, 92 \%$, respectively. A serial combination (100 keV VMI followed by $\mathrm{Z}$ effective images) resulted in an improved specificity with a moderate loss of sensitivity. An illustrative example from their paper is shown below, where the significant decrease in the blooming is very well visible, and where the percent narrowings very well match.

Johan H. C. Reiber

J.H.C.Reiber@lumc.nl

1 Leiden, The Netherlands 


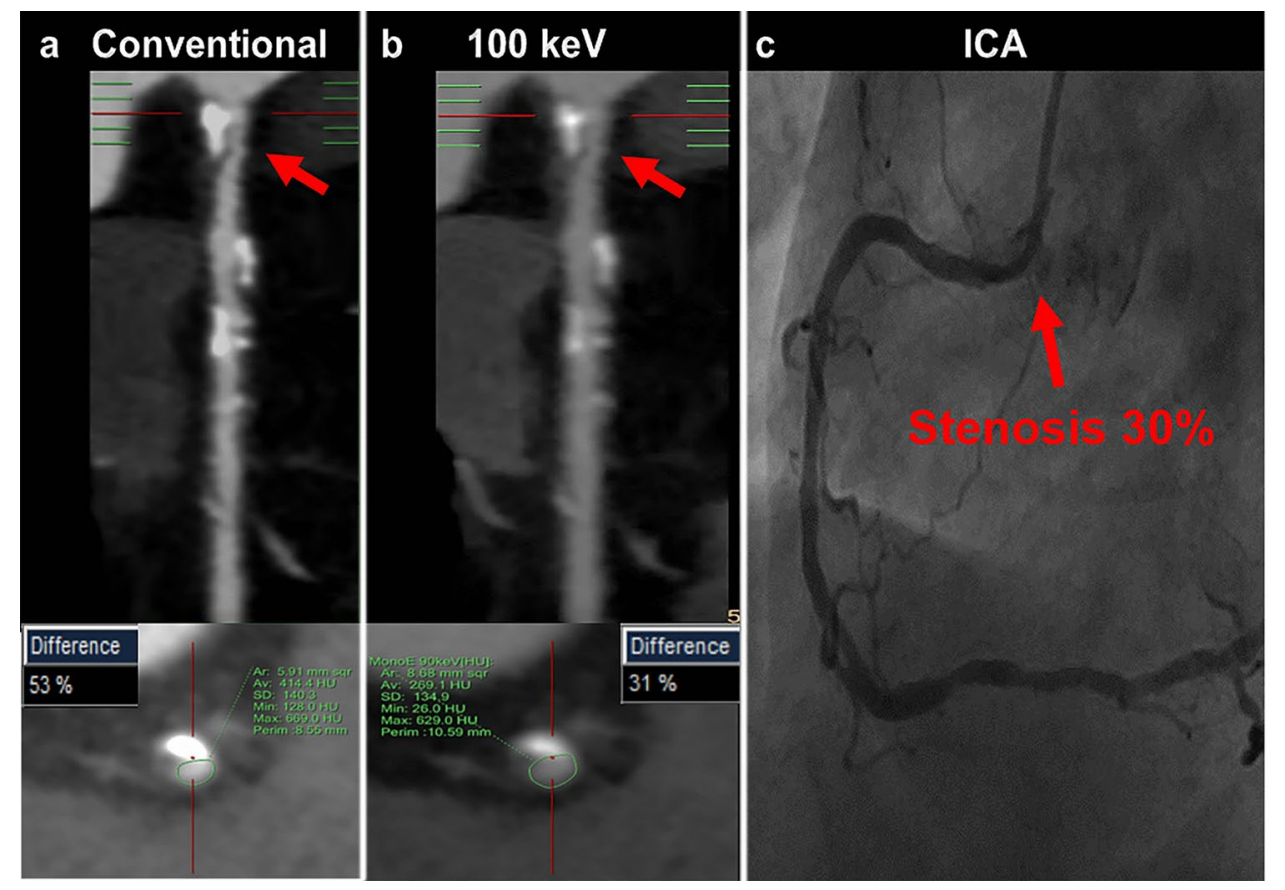

The authors describe also a number of limitations of this study with a small sample size, and also that retrospective ECG gating protocol was used in this study, and the radiation dose was relatively high, which is of course extremely important, if this wants to become a standard practice. But the first results are encouraging.

The developments described in this manuscript are by Philips Medical, but of course all MSCT companies will come up with their own solutions, which should lead to a better visualization of coronary and peripheral vessels, as well as valves, which also have significant amounts of calcium. For further details about this particular work, I refer the readers to the full paper in this issue of the International Journal of Cardiovascular Images.

Johan H. C. Reiber, Ph.D.

Editor-in-chief

\section{Reference}

1. Xu C, Yi Y, Han Y et al (2021) Incremental improvement of diagnostic performance of coronary CT angiography for the assessment of coronary stenosis in the presence of calcium using a dual-layer spectral detector CT: validation by invasive coronary angiography. Int J Cardiovasc Imaging. https://doi.org/10.1007/ s10554-021-02205-3

Publisher's Note Springer Nature remains neutral with regard to jurisdictional claims in published maps and institutional affiliations. 